\title{
A long term follow up of results of women undergoing an office based thermablate endometrial ablations for the treatment of menorrhagia
}

\begin{abstract}
Background: Heavy menstrual bleeding (HMB) or menorrhagia accounts for $20 \%$ of outpatient referrals to secondary gynaecological services. The Thermablate Endometrial Ablations system (EAS) is an example of a second generation of EAS used for treating menorrhagia of benign origin; reducing the necessity for a hysterectomy. The study aimed to explore long term results and success of this system's use in an outpatient setting.

Methods: This prospective observational study examined the long term follow up of 175 women attending an outpatient setting, having a Thermablate EA between July 2008 and July 2013. Women included had menorrhagia unresponsive to medical or hormonal therapy; and demonstrated a normal uterine cavity on hysteroscopy. They all received oral analgesia preceding ablation and welfare was ensured post procedure. Final outcomes were gathered using NOTIS; determining intervention rates following Thermablate EAS and satisfaction and improvement of quality of life through minimisation of bleeding and better symptom control.

Results: Of the 173 women, 93 women (53.7\%) had lighter menstrual periods and 45 women $(26 \%)$ became amenorrhoeic. 138 women $(79.7 \%)$ had no interventions following Thermablate EAS. Our complication rate was $1.2 \% .11$ women went on to have hysterectomy for persistent uterine bleeding.

Key message: Thermablate EAS is an extremely well tolerated device ideal for use in the outpatient office setting. The long term results demonstrate high satisfaction rates and better symptom control acceptable to most women. Low intervention rate demonstrated in the form of hysterectomy has important cost implications for the NHS.
\end{abstract}

Volume 4 Issue 5 - 2016

\author{
Atia Qaiser, Chen BF, Powell MC \\ Kings Mill Hospital, UK
}

Correspondence: Atia Qaiser, Kings Mill Hospital, Mansfield Road, Sutton-in-Ash field, Nottinghamshire, NG 17 4JL, UK, Tel 74|47674I2, Email atiaqaiser@doctors.org.uk

Received: May 05, 2016 | Published: June 21, 2016

\section{Introduction}

Heavy menstrual bleeding (HMB) or menorrhagia, is defined as menstrual blood loss lasting more than 7 days over consecutive cycles, or more than $80 \mathrm{ml}$ per cycle. ${ }^{1}$ This a common problem amongst premenopausal women. Such excessive loss can lead to detrimental consequences affecting all aspects of a woman's life. Their physical health can be affected as a result of iron deficiency anaemia. HMB can also have effects on daily activities such as employment or socialising; through to the implications one motional quality of life with issues of embarrassment, depression and problems with their sex lives. ${ }^{2}$

It is a common gynaecological condition affecting upto $30 \%$ of women during their reproductive years and accounts for $20 \%$ of out patient referrals to secondary gynaecological services. ${ }^{2}$ In the UK, it is likely that one in five women will undergo a hysterectomy by the age of 55years. ${ }^{3}$ Although a definitive treatment resulting in a menorrhea, it is not without significant risk. It has the mortality of 0.4-1.1 per 1000 operations; as well as major complications in 1 in 30 women, including anaesthetic problems, haemorrhage, pulmonary embolism, urinary retention and peripheral nerve injuries. In addition, many psychological issues can ensue such as loss of femininity and depression. ${ }^{4}$ Of those women being treated for menorrhagia, as many as half can be found to have a normal uterus. ${ }^{5}$

In light of this evidence, current guidelines ${ }^{1,6}$ recommend exploring a more conservative approach initially through less invasive procedures such as endometrial lablation. The Thermablate
Endometrial Ablations system (EAS) is one such example of a second generation of EAS used for treating menorrhagia of benign origin. ${ }^{7}$

The literature has already suggested that this method is effective in achieving a menorrhea or lighter periods and so reducing the necessity for a hysterectomy. ${ }^{8}$ The aim of this study was to explore the long term results and success of this system's use in an outpatient setting.

\section{Methods}

This prospective observational study looked at the long term follow up of 175 women attending the Ambulatory Menstrual Disorders Clinic at the Nottingham Circle Treatment Centre. These women had a Thermablate EAS between July 2008 and July 2013.

\section{Patients}

The inclusion criteria were defined as women identified as having menorrhagia and a demonstration of unresponsiveness to conservative medical or hormonal therapy. All women were demonstrated to have a normal uterine cavity on a diagnostic hysteroscopy and endometrial biopsy and a cervical to uterine fungal length between 7-14centimetres (cm). Hysteroscopy was always repeated post ablation to inspect the uterine cavity to demonstrate the integrity of the uterus and the result to the patient.

Women with malignant lesions, submucous fibroids greater than $3 \mathrm{~cm}$ or a history of more than three cases are an section were excluded. All women were counseled about the use of reliable contraception and 
that the desire to conserve fertility would be a contraindication for the use of Thermablate EAS. The women understood the procedure would be performed under a local anaesthetic in the office setting. All procedures were conducted by the same surgeon, with the assistance of two nurses.

All women had oral analgesia 2 hours preceding the Thermablate EAS in the form of 100milligrams (mg) diclofenac and $50 \mathrm{mg}$ tramadol. A perioperative intracervical block of $4 \%$ prilocaine and intracavitary 6 millilitres $(\mathrm{ml})$ of $2 \%$ lidocaine were also administered. The women were discharged from the clinic post procedure usually within 30minutes, provided they felt well and had achieved adequate pain relief. They were discharged with a supply of oral analgesia. All women received a nurse telephone consultation the day following their procedure to highlight any concerns with follow up in the outpatients clinic at 4 months.

Final out comes were gathered using NOTIS, the hospital patient data system; to establish bleeding patterns and any further interventions required. Ethical approval was not required as the data was collected anonymously with no patients' personal data.

The primary outcome of this study was to examine the long-term results of this office based, local anaesthetic Thermablate EAS in the treatment of menorrhagia. In achieving this we also examined the intervention rates following Thermablate EAS and satisfaction and improvement of quality of life through minimization of bleeding and better symptom control.

\section{Results}

175 women underwent a Thermablate ablation. 2 patients did not attend their follow-up appointment and hence, were excluded from this study although no record was found on NOTIS of subsequent intervention. 20 women did not require any follow-up the procedure. All women had a telephone consultation the day after and were given contact details in case of any questions or problems encountered.

Of the 173women, 93women (53.7\%) had lighter menstrual periods and 45 women $(26 \%)$ became amenorrhoeic. However, 35 women complained of unacceptable bleeding $(20.2 \%)$, of whom $2.3 \%$ continued to have on-going bleeding and pelvic pain. 2 women additionally had symptoms of pelvic pain despite havings at is faction in their menstrual period (Figure 1).

Our complication rate was $1.2 \%$. Two women had a post-operative infection, where one of them developed a haematometra. One woman became pregnant after the ablation and had a successful full- term pregnancy.

138 women $(79.7 \%)$ had no interventions following Thermablate EAS. 2 women (1.2\%) had non-hormonal conservative treatment, i.e. analgesia and counselling. 6 women(3.5\%) had hormonal medical treatment (oral nor-ethisterone, or Gonadotrophin-releasing hormone (GnRH) analogues). 27 women into tal had surgical interventions including diagnostic laparoscopy, laparoscopic drainage of ovarian cyst, hysteroscopy, hysteroscopic drainage of haematometra, transcervical resection of fibroid (TCRF) and trans-cervical resection of endometrium (TCRE) (Figure 2). In this group of women, 11 women went on to have hysterectomy for persistent uterine bleeding. None had a hysterectomy for abnormal endometrial pathology. The group shown to have no further interventions had a mean follow up time of 214 days. Those women requiring non-hormonal conservative treatment required the least follow up, compared to those that went on to have hormonal treatment who required the longest duration (Figure $3)$.

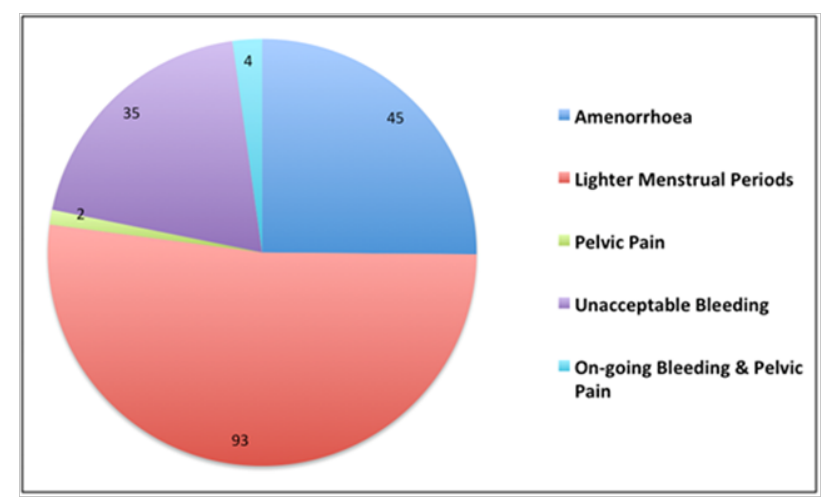

Figure I Thermablate EAS Symptom Outcomes (no of women).

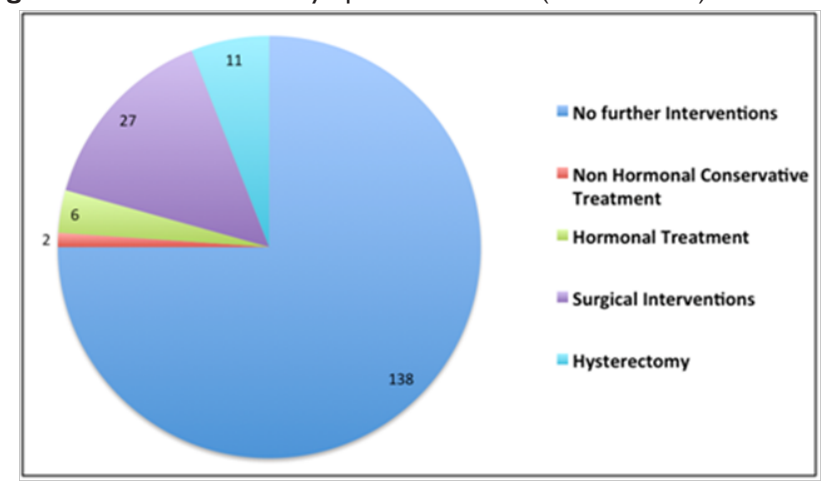

Figure 2 Interventions following Thermablate Ablations (no of women).

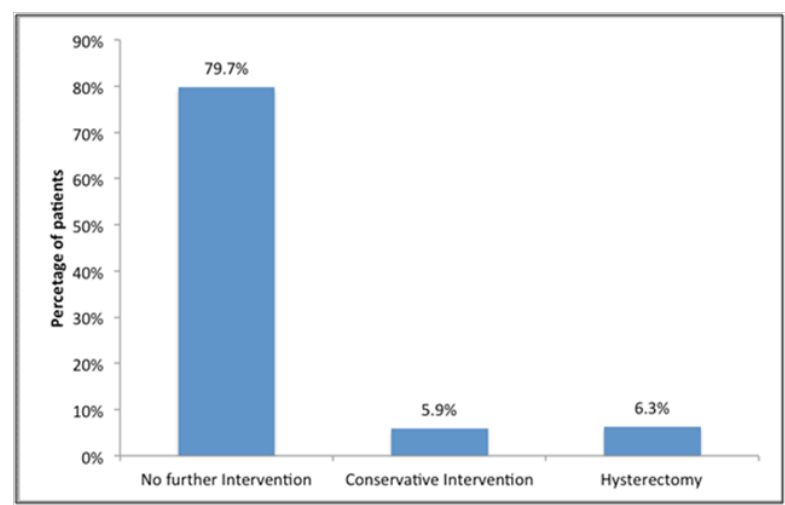

Figure 3 Interventions following Thermablate EA

\section{Discussion}

The TEAS provides a valid and effective alternative therapy in those women where other choices are either contra indicated or would prove technically challenging due to an irregular uterine cavity. ${ }^{9} 10$

It is a light weight device that employs are usable hand-held treatment control unit with a disposable fluid-filled balloon catheter system. An automatic treatment cycle involves inflation and eflation of the $13 \mathrm{ml}$ balloon catheter over 2 minutes. Uniform destruction of the myometrium is facilitated by the therapeutic high temperature $\left(173^{\circ}\right.$ Celsius $)$ and pressure $(220$ millimeter of mercury $(\mathrm{mmHg}))$ employed. Our experience has demonstrated that the procedure is simple to perform. The short operation time and narrow catheter facilitate this, allowing it to be well tolerated. In another prospective observational study performed in our centre, Thermablate EAS appeared to be well- accepted and safe procedure for treating menorrhagia. 
Thermablate EAS has not been as extensively reported as other balloon thermal ablation. The available studies have been in small numbers ranging from 16 to 90 patients and shorter follow- up of 6 months and up to 2 years. ${ }^{11-14}$ Amenorrhoea rates of $25 \%$ were reported, with success rates of $80 \%$. No major complications were reported in either study. ${ }^{11,12}$ Our study of a larger population demonstrates a menorrhoea rates of $26.0 \%$ and satisfaction around $80 \%$. We have as lighter higher complication rate of $1.2 \%$ as compared to other studies. ${ }^{11-14}$ This can be due to the higher number of cases in our studies.

There can be other cause of menorrhagia in women over 40years old other than dysfunctional uterine bleeding. Therefore, in our unit, we ensure that all the women had an endometrial biopsy prior to the TEAS treatment. Further, during hysteroscopy prior to the treatment, the endometrium is assessed for abnormal appearance before proceeding. Out of all our women, none had any abnormal uterine pathology. Other study had similar out comes with their endometrial pathology of $1.7 \%{ }^{14}$ (Figure 4).

Of the women that were treated $5 \%$ of women classified themselves from ethnic minorities with the remaining demographic of $95 \%$ classifying themselves as White British. This representation of ethnic minorities is slightly less than the national average figures in the $\mathrm{UK}^{15}$ (Table 1).

National Institute for Health and Clinical Excellence (NICE) estimates that if all hysterectomies were to be replaced by endometrial ablation using second-generation devices, the NHS would save $£ 32$ million per year. NICE concedes that his assumption is simplistic, as some women will in variably need or prefer hysterectomy. ${ }^{16}$ Although hysterectomy is associated with longer operating time (particularly for the laparoscopic route), a longer recovery period and higher rates of postoperative complications, it offers permanent relief from heavy menstrual bleeding. Endometrial ablation offers an alternative to hysterectomy as a surgical treatment for heavy menstrual bleeding. It is effective, and satisfaction rates are high ${ }^{17}$ (Table 2).

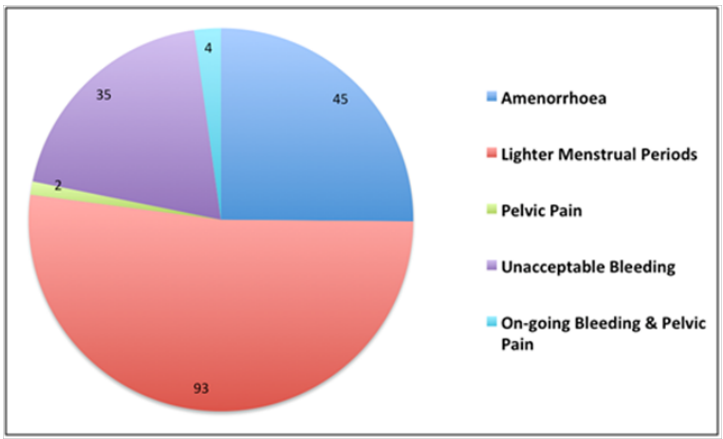

Figure 4 Ages of women demonstrating resolution of symptoms compared to those with no improvement.

Table I Patient demographics including ethnicity

\begin{tabular}{ll}
\hline Parameter & No. of women \\
\hline Age(Year); mean(range) & $47(30-59)$ \\
Ethnicity & 175 \\
White British & 164 \\
Black Caribbean & 2 \\
Indian & 2 \\
Pakistani & $\mathrm{I}$ \\
Mixed:White \& Black Caribbean & 2 \\
Any other Mixed background & $\mathrm{I}$ \\
Any other Asian background & $\mathrm{I}$ \\
\hline
\end{tabular}

Table 2 Mean followup duration

\begin{tabular}{ll}
\hline Parameter & No. of days \\
\hline No further intervention & 214 \\
Amenorrhoeic & 117 \\
Lighter menstrual bleeding & 267 \\
Non hormonal conservative treatment & 236 \\
Hormonal treatment & 664 \\
Surgical intervention & 492 \\
Hysterectomy & 403
\end{tabular}

\section{Conclusion}

Thermablate EAS is an extremely well tolerated device that is ideal for use in the outpatient office setting. The long term results demonstrate high satisfaction rates and better symptom control acceptable to most women. We have also demonstrated the low intervention rate in the form of hysterectomy, which has important cost implications for the NHS.

\section{Acknowledgments}

None.

\section{Conflicts of interest}

None.

\section{References}

1. National Collaborating Centre for Women's and Children's Health, National Institute for Health and Clinical Excellence. Heavy menstrual bleeding. Clinical Guideline No.44. NICE, London. 2007.

2. RCOG. National Heavy Menstrual Bleeding Audit: A national audit to assess patient outcomes and experiences of care for women with heavy menstrual bleeding in England and Wales. First annual report. 2011;p:1-68.

3. Vessey MP, Villard-Mackintosh L, Mc Pherson K, et al. The epidemiology of hysterectomy: findings in a large cohort study. $\mathrm{Br} J$ Obstet Gynaecol. 1992;99(5):402-407.

4. Coulter A, Mc Pherson K, Vessey M. Do British women undergo too many or too few hysterectomies? Soc Sci Med. 1988;27(9):987-994.

5. Maresh MJ, Metcalfe MA, McPherson K, et al. The VALUE national hysterectomy study: description of the patients and their surgery. BJOG. 2002;109(3):302-312.

6. NICE. Fluid filled thermal balloon and microwave endometrial ablation techniques for heavy menstrual bleeding. National Institute of Health and Clinical Excellence guidelines. 2004.

7. Neuwirth RS, Duran AA, Singer A. The endometrial ablator: a new instrument. Obstet Gynecol. 1994;83:792-796.

8. Daniels JP, Middleton LJ, Champaneria R, et al. Second generation endometrial ablation techniques for heavy menstrual bleeding: network meta-analysis. BMJ. 2012;344:e2564.

9. Prasad P, Powell MC. Prospective Observational Study of Thermablate Endometrial Ablation Systemasan Outpatient Procedure. J Minim Invas Gyn. 2008;15(4):476-479.

10. Karamanidis D, Nicolaou P, Byros A, et al. Two-year results of a new two-minute hot liquid balloon endometrial ablation system (Thermablate): a pilot study. Clin Exp Obstet Gynecol. 2009;36(4):256258

11. Mangeshikar PS, Kapur A, Yackel DB. Endometrial ablation with a new thermal balloon system. J Am Assoc Gynecol Laparosc. 2003;10(1):2732 . 
12. Douglass B, Yackel DB, Vilos G. Thermablate EAS: a new endometrial ablation device. Gynecol. 2004;Surg1(2):129-132.

13. Justin W, Ibraheim M, Bagtharia S, et al. Current minimal access techniques in the treatment of heavy menstrual bleeding. The Obstetrician \& Gynaecologist. 2007;9(4):223-232.

14. Hou X, Feng L. Long-term efficacy analysis of abnormal uterine bleeding treated with thermablate TMEASTM. Journal of Minimally Invasive Gynecology. 2014;21(6):S143.
15. Ethnicity and National Identity in England and Wales: 2011. Office for National Statistics. 2012

16. National Institute of Health and Clinical Excellence. Menstrual Bleeding-Fluid-filled Thermal Balloon and Microwave Endometrial Ablation. Technology Appraisal 78. NICE, London. 2004.

17. Lethaby A, Shepperd S, Cooke I, et al. Endometrial resection and ablation versus hysterectomy for heavy menstrual bleeding. Cochrane Database Syst Rev. 1999;(2):CD000329. 